\title{
How to Manage a Failed Cartilage Repair: A Systematic Literature Review
}

\author{
Donato Rosa ${ }^{1}$ Sigismondo Luca Di Donato ${ }^{1}$ Giovanni Balato ${ }^{1} \quad$ Alessio D’Addona ${ }^{1}$ \\ Francesco Smeraglia ${ }^{1}$ Gaetano Correra ${ }^{1}$ Gianni Di Vico ${ }^{2}$ \\ 1 Orthopaedic Unit, Department of Public Health, School of Medicine \\ "Federico II" University, Naples, Italy \\ 2 Department of Orthopaedic Surgery, "S. Michele" Clinic, \\ Maddaloni, Italy \\ Address for correspondence Sigismondo Luca Di Donato, MD, \\ Orthopaedic Unit, Department of Public Health, School of Medicine \\ “Federico II" University, Via Sergio Pansini 5, Building 12, 80131, \\ Naples, Italy (e-mail: sigismondodidonato@gmail.com).
}

Joints 2017;5:93-106.

\begin{abstract}
Purpose The aims of this paper are to report the rate and risk factors for the failure of the most common cartilage repair technique, and analyze the most important factors that could influence the choice of a specific surgical treatment to revise a failed cartilage repair.

Methods A review of the literature was performed focusing on failed cartilage repair and related treatments. Two of the authors independently screened articles. Conflicts about the inclusion of a paper was resolved by further evaluation by the senior author. Review articles, articles written in languages different from/other than English, case reports, and papers that did not evaluate the outcomes of interest were excluded. Fulltext version of each included paper was obtained and relevant data were extracted and collected in a database.

Results At the end of the screening process, 31 articles were included. Microfractures and mosaicplasty showed a nonnegligible failure rate at short- and midterm. Better results, especially in terms of time to failure or revision, were reported with the use of autologous chondrocyte implantation $(\mathrm{ACl})$ and osteochondral allograft (OCA) transplantation. Regarding the treatment of failed cartilage repair, the use of OCA transplantation in patients with previous failed cartilage repair may be a safe option. The revision of failed OCA transplantation with further OCA seems to have a greater failure rate. Patients with previous failed $\mathrm{ACl}$ or matrix-induced autologous chondrocyte implantation (MACl) who underwent further $\mathrm{MACl}$ or $\mathrm{ACl}$ reported acceptable

Keywords

- cartilage

- repair

- failure

- revision

- surgery results. Otherwise, $\mathrm{ACl}$ in patients with history of previous subchondral marrow stimulation (SMS) demonstrated a greater failure rate.

Conclusion From the analysis of the literature, OCA transplantation seems to be the most reliable treatment of a failed SMS. ACl or MACl showed acceptable results in patients with previously failed $\mathrm{MACl}$ or $\mathrm{ACl}$.

Level of Evidence Level IV, systematic review of level I-IV studies.
\end{abstract}

\section{Introduction}

Cartilage defects are very common lesions reported in $63 \%$ of patients who underwent arthroscopy. ${ }^{1}$ Actually, there are different surgical techniques of cartilage repair, such as autologous chondrocyte implantation (ACI), subchondral marrow stimulation (SMS), osteochondral autograft transplantation (OAT), and osteochondral allograft (OCA) transplantation. In literature, different algorithms of treatment
DOI https://doi.org/ 10.1055/s-0037-1603900. ISSN 2282-4324.
Copyright (c) 2017 Georg Thieme Verlag License terms KG Stuttgart · New York 
are proposed. ${ }^{2,3}$ Compared with treatment of cartilage defects in general, there is a lack of published information about the management of failed cartilage repair. In addition, only in the last years, the attention on the effect of a previous cartilage surgery on a subsequent revision cartilage restoration has increased. The aims of the present review are to report the rate and risk factors for failure of most common surgical repair techniques in general population and in patients with a previous failed cartilage repair, and highlight the most important factors that could influence the choice of a specific surgical treatment to revise a failed cartilage repair.

\section{Methods}

To identify relevant papers dealing with failures of cartilage repair of the knee and their surgical management, we performed a literature research on failures of cartilage repair in English language using the PubMed database with the search entries "failure" OR "failed" AND "reoperation" OR "revision" AND "cartilage" AND "knee." Two hundred fiftythree papers, published in the last 10 years, were extracted. Two authors independently reviewed each title and abstract. Once a paper was identified as likely to be included, full-text versions were obtained. Conflict about the inclusion of a paper was resolved by further evaluation by the senior author. Two hundred twenty-two papers were excluded from the analysis for the following reasons: 209 did not evaluate the outcomes of interest, 6 were review articles, 1 was not in English, and 6 were case reports. Therefore, 31 papers were finally included (-Fig. 1 ). These papers are summarized in -Table 1.

\section{Results}

\section{Microfracture}

Salzmann et $\mathrm{al}^{4}$ conducted a retrospective study on a large cohort of 454 patients (mean defect size, $2.97 \mathrm{~cm}^{2}$ ) treated by

\section{Articles screened}

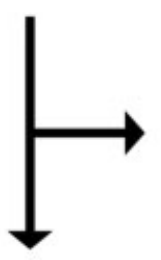

217 studies excluded after Title/Abstract reviewed: 206 studies not evaluated the outcome of interest; 4 reviews; 6 case reports, 1 non-English language.

36 Full-text studies reviewed

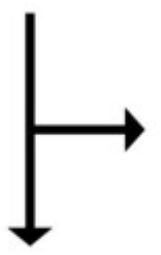

5 studies excluded: 3 studies not evaluated the outcome of interest; 2 reviews.

\section{Studies were included}

Fig. 1 Flow chart of studies identified and selected for the review. microfracture (MF). In this study, patients that underwent revision surgery at the index knee joint with pain related to the initial surgical site were described as "failure." Subjects with history of any trauma leading to reoperation at the index knee following the initial MF were excluded. The failure rate was $27.1 \%$ (123 patients) and the mean time between initial MF and reoperation was 1.6 years. Failure subjects had significantly more previous surgeries to the index knee, and interestingly, significantly smaller total defect dimensions/knee, and a smaller dimension of the largest lesion of the knee joint.

Solheim et $\mathrm{al}^{5}$ prospectively collected data of approximately 110 patients who underwent MF (median defect size, $4 \mathrm{~cm}^{2}$ ). The authors defined a case as failure that underwent a new surgical procedure to treat the cartilage lesion. The authors reported 24 failures (21.82\%) in the study population. Steadman et $\mathrm{al}^{6}$ studied a specific population of 26 patients younger than 19 years (mean defect size, $1.77 \mathrm{~cm}^{2}$ ) treated with MF. In this study, 2-year follow-up was obtained in 22 of 26 patients with an average of 5.8 years. Failure criteria were not specified. The authors reported that three patients had Lysholm scores of less than 80 at a minimum of 2 years after MF surgery. All three patients were females and 18 years old. Two underwent MF of the patella and one underwent MF of the trochlear groove. The patient who underwent trochlear groove MF had a revision MF after 1 year from the initial MF procedure. No other patient required revision.

\section{Osteochondral Autograft Transplantation}

In a long-term (minimum 10 years of follow-up) randomized controlled trial (RCT), Bentley et $\mathrm{al}^{7}$ compared ACI versus mosaicplasty. One hundred consecutive patients with a symptomatic articular cartilage lesion were randomized to undergo either $\mathrm{ACI}$ or mosaicplasty. The failure criteria adopted by the authors were clinically poor results with arthroscopic evidence of failure of the graft, or revision surgery for any kind of defect. Forty-two patients (mean defect size, $4 \mathrm{~cm}^{2}$ ) were enrolled in the group treated by mosaicplasty. Among this group, a high failure rate was recorded ( 23 out of 42 patients, 55\%), with a mean time from the index procedure to revision surgery of 4.3 years. Compared with $\mathrm{ACI}$, mosaicplasty showed a statistically significant higher number of failures $(p<0.001)$. The authors declared that the mosaicplasty group remained relatively satisfactory for the first 2 years, and then experienced a steep failure rate over the next 2 years. Of the 23 failed patients, 9 underwent revision surgery with $\mathrm{ACI}, 3$ were revised with matrix-induced autologous chondrocyte implantation (MACI), 3 were converted to unicompartmental knee replacement (UKR), 1 to total knee replacement (TKR), 1 to patellofemoral joint (PFJ) replacement, and 1 to combined medial and PFJ replacement. Five patients were classified as unknown or revised elsewhere.

Robb et $\mathrm{al}^{8}$ evaluated 55 patients (mean defect size, $2.2 \mathrm{~cm}^{2}$ ) who underwent OAT (mosaicplasty) with a mean follow-up of 5.9 years. The following were considered as failures by the authors: degeneration of the articular surface, 
Failed Cartilage Repair Rosa et al.

95

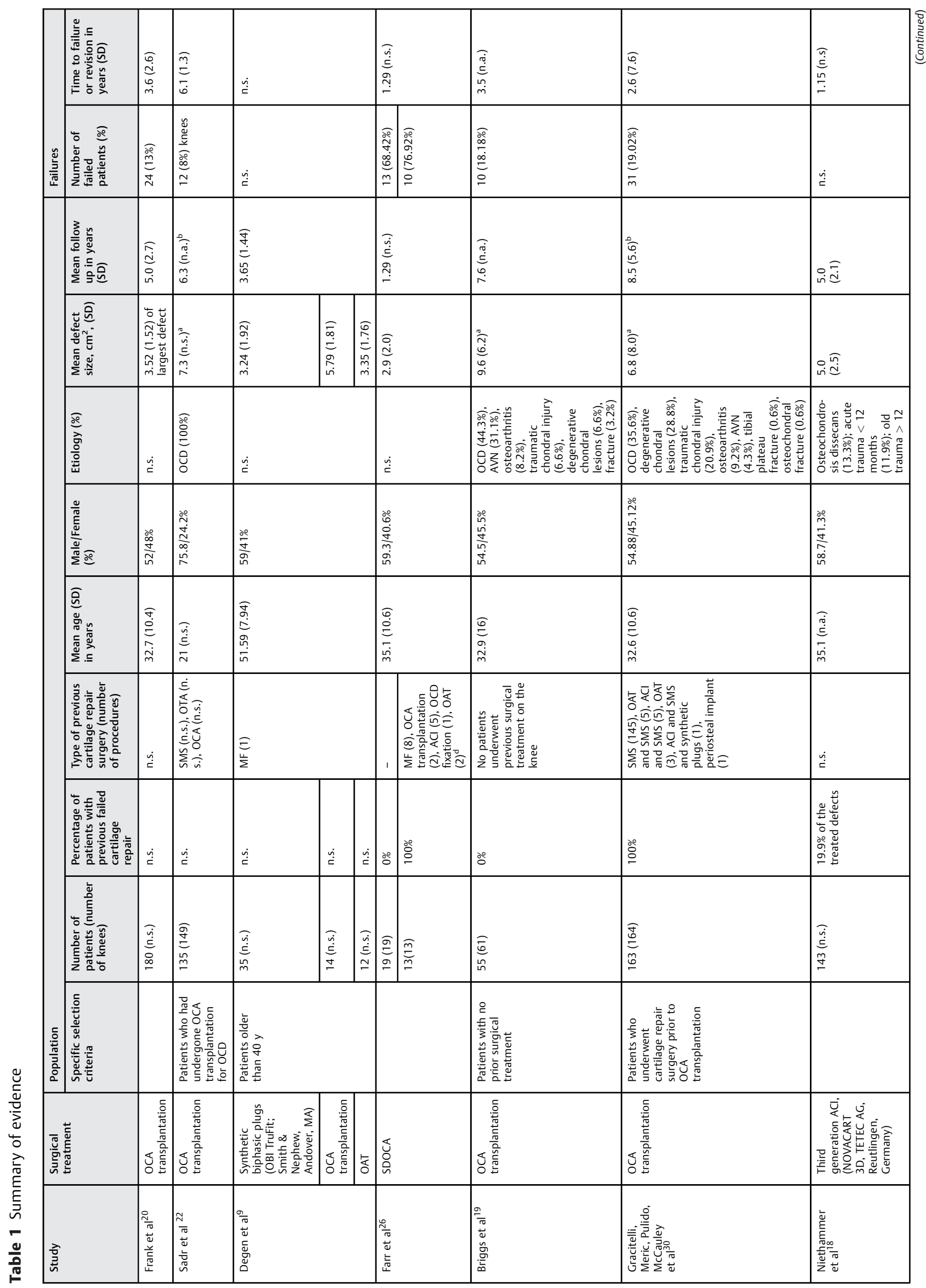


Failed Cartilage Repair Rosa et al.

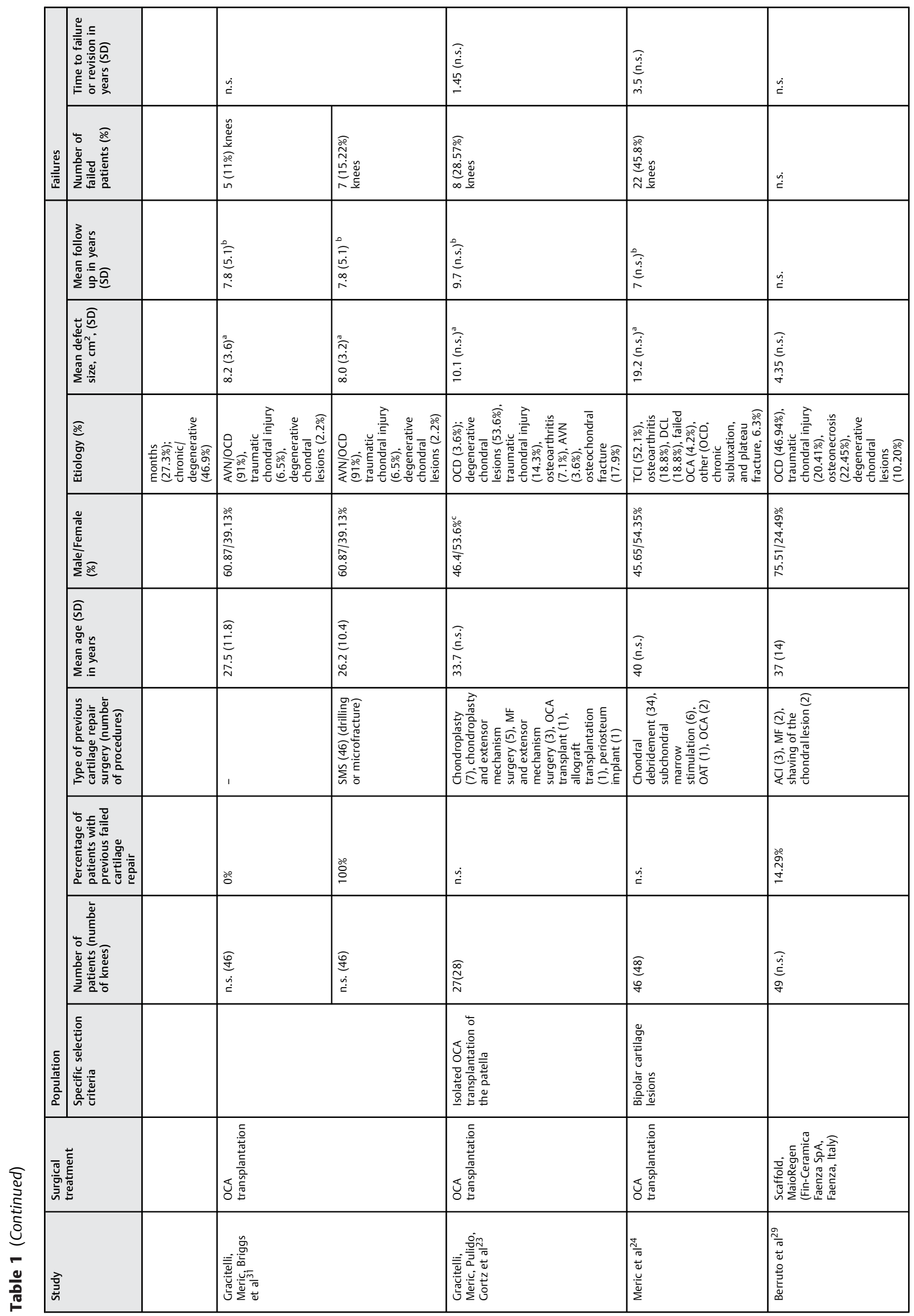


Failed Cartilage Repair Rosa et al.

97

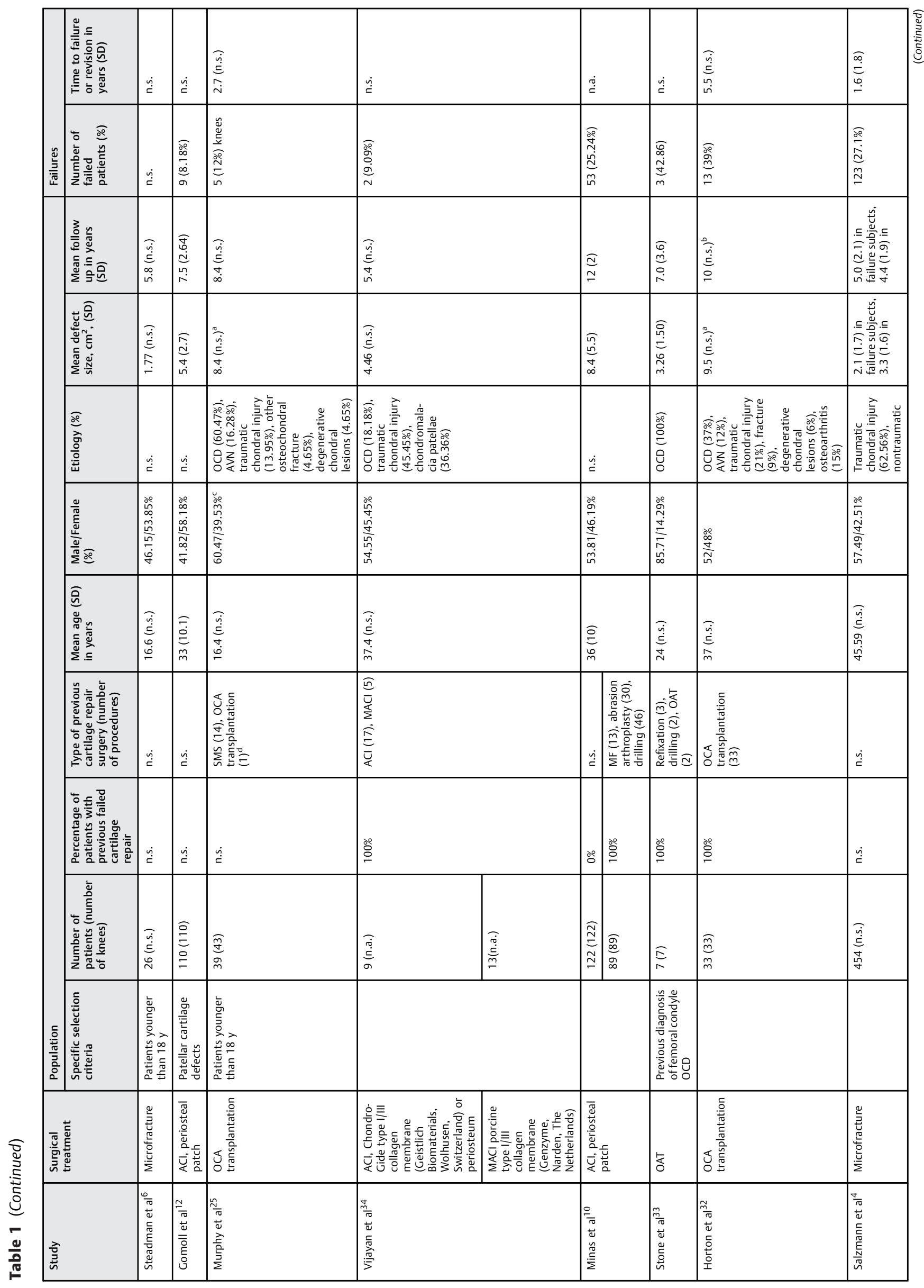


Failed Cartilage Repair Rosa et al.

\begin{tabular}{|c|c|c|c|c|c|c|c|c|}
\hline 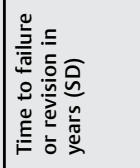 & & 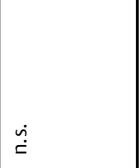 & \begin{tabular}{|l}
$\tilde{a}$ \\
$\underline{n}$ \\
\\
\end{tabular} & \begin{tabular}{|l}
$\dot{g}$ \\
$\check{c}$
\end{tabular} & 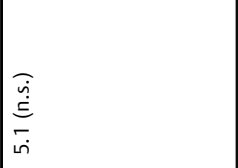 & 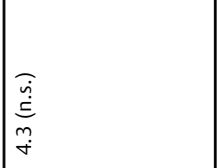 & 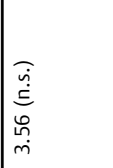 & 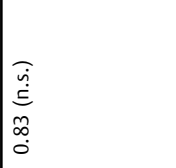 \\
\hline 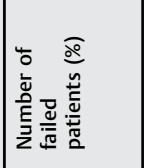 & & 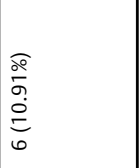 & 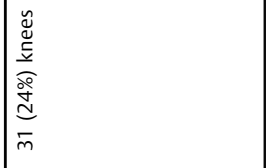 & $\dot{\stackrel{\dot{g}}{\check{c}}}$ & 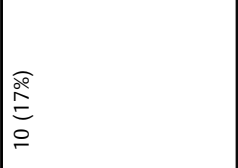 & 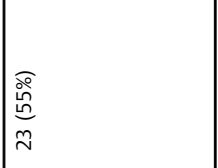 & $\mid \begin{array}{l}\dot{\varphi} \\
\check{c}\end{array}$ & $\begin{array}{l}\dot{\dot{g}} \\
\check{c}\end{array}$ \\
\hline 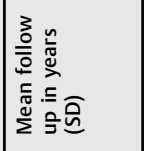 & 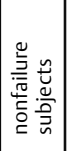 & 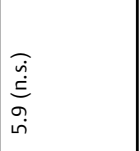 & 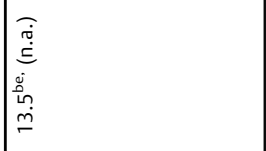 & 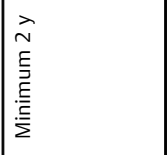 & 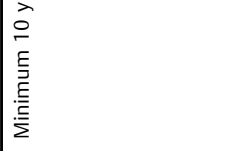 & & $\mid \begin{array}{l}\overline{\hat{g}} \\
\infty \\
\infty\end{array}$ & $\mid \stackrel{\dot{\varphi}}{\check{c}}$ \\
\hline 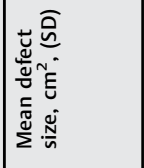 & 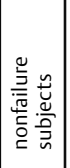 & $\begin{array}{l}\overline{\tilde{u}} \\
\underset{\tilde{S}}{\tilde{N}}\end{array}$ & 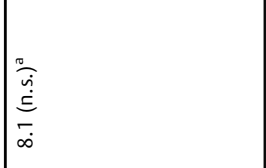 & 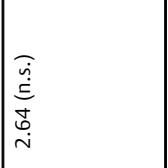 & 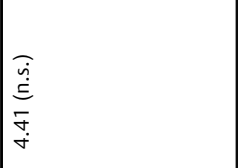 & $\mid \begin{array}{c}\overline{\dot{\varphi}} \\
\stackrel{\dot{\sigma}}{\sigma}\end{array}$ & 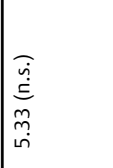 & 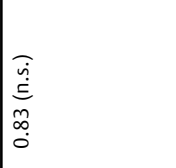 \\
\hline 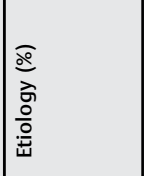 & 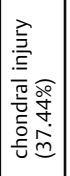 & 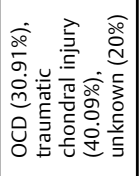 & 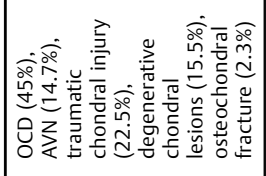 & 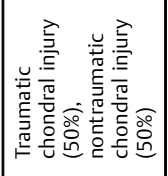 & 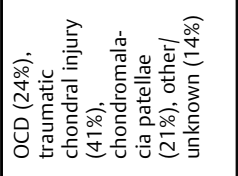 & 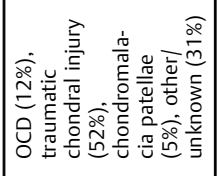 & 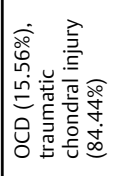 & 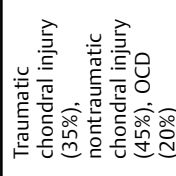 \\
\hline 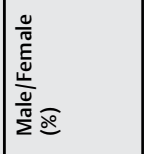 & & 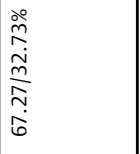 & $\begin{array}{l}\frac{\partial}{\partial} \\
\frac{\partial}{N} \\
\end{array}$ & 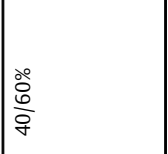 & $\begin{array}{l}\stackrel{\circ}{2} \\
\frac{2}{\infty} \\
\text { in }\end{array}$ & & 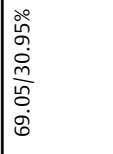 & 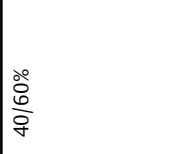 \\
\hline 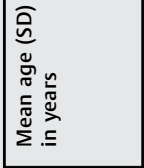 & & 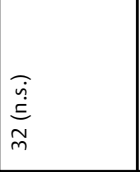 & 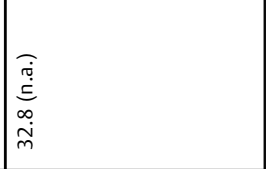 & 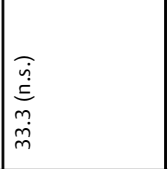 & 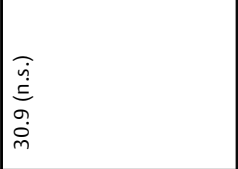 & 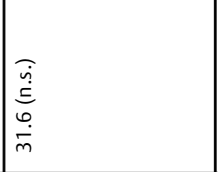 & 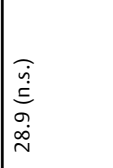 & 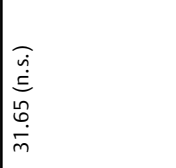 \\
\hline 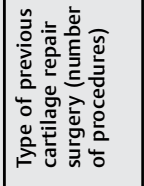 & & $\begin{array}{l}\bar{E} \\
\bar{\psi}\end{array}$ & 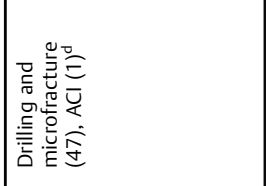 & $\mid \stackrel{\dot{a}}{\check{c}}$ & 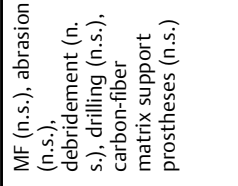 & & $\dot{\stackrel{\dot{g}}{i}}$ & 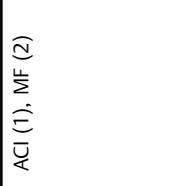 \\
\hline 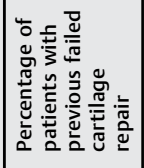 & & ֻْ & $\mid \stackrel{\dot{S}}{\check{\Sigma}}$ & $\dot{\stackrel{\dot{a}}{e}}$ & $\stackrel{\circ}{6}$ & & $\dot{\stackrel{\dot{g}}{e}}$ & $\dot{\leftrightarrow}$ \\
\hline 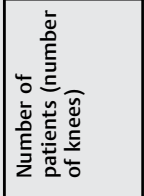 & & 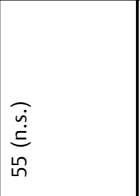 & 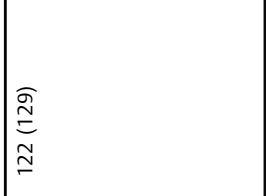 & 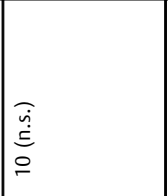 & \begin{tabular}{|l}
$\overline{\tilde{u}}$ \\
$\stackrel{\tilde{\omega}}{\infty}$ \\
$i n$
\end{tabular} & $\begin{array}{l}\widehat{\widetilde{\varphi}} \\
\dot{S} \\
\tilde{\gamma}\end{array}$ & $\begin{array}{l}\frac{\pi}{\partial} \\
\tilde{\gamma}\end{array}$ & i \\
\hline 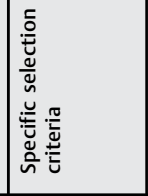 & & & 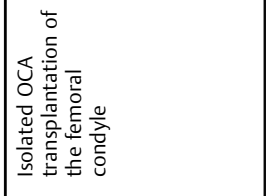 & 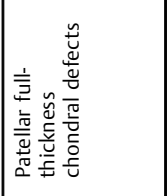 & & & & \\
\hline & & 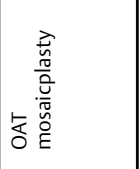 & 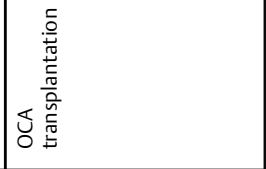 & 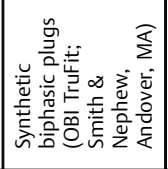 & 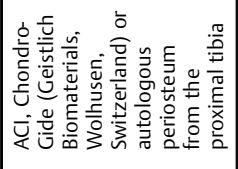 & 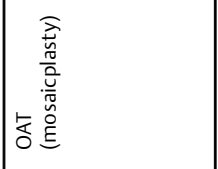 & 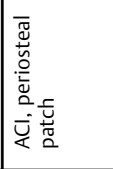 & 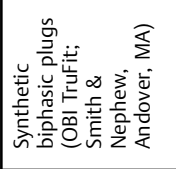 \\
\hline & & 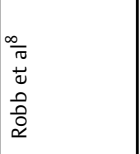 & 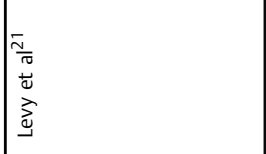 & $\mid$ & $\mid$ & & 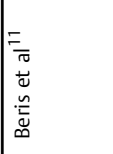 & 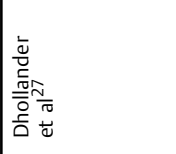 \\
\hline
\end{tabular}




loosening of the OAT graft, any subsequent osteochondral regeneration or reconstruction procedure, limb realignment surgery, any form of arthroplasty, or a poor Oxford Knee Score (OKS). Six failed patients (10.91\%) were reported, four of these were due to revision surgery and two due to a poor OKS. Of the four patients who required revision surgery, two had a medially localized cartilage lesion and varus malalignment. One of these two patients had revision OAT and a high tibial osteotomy (HTO); the other underwent UKR. The other two patients who required revision surgery were treated respectively with TKR and revision OAT.

Degen et $\mathrm{al}^{9}$ compared three groups of patients older than 40 years (mean follow-up, 3.65 years) with symptomatic distal femoral cartilage lesion, and respectively treated with synthetic scaffold plugs (OBI TruFit; Smith \& Nephew, Andover, Massachusetts, United States), OCA transplantation and OAT (mosaicplasty). Patients with adjacent untreated regions in the operative knees worse than Outerbridge grade 2 changes or bipolar tibiofemoral lesions requiring treatment of the tibial lesion were excluded. No failure criteria were established. Twelve patients (mean defect size, $3.35 \mathrm{~cm}^{2}$ ) were included in the OAT or mosaicplasty group, and among these patients, only 1 subject required arthroscopic debridement, lysis of adhesions, and removal of loose bodies at 1.25 years.

\section{Autologous Chondrocyte Implantation}

Minas et $\mathrm{al}^{10}$ analyzed a population of 210 patients (total surface area treated per knee, $8.4 \mathrm{~cm}^{2}$ ) treated with periosteum patch-covered ACI for symptomatic full-thickness chondral defects of the knee and a minimum follow-up of 10 years. The authors categorized the failures as graft failure with revision using partial knee arthroplasty or TKA; graft failure with revision cartilage repair; and graft survival but development of new defects elsewhere in the same knee requiring additional surgery. The failure rate was $25 \%$ (53 patients). After failure, 19 patients underwent knee arthroplasty, cartilage repair procedures were performed in 27 patients, and 7 patients declined further treatment. An analysis of possible risk factors for failure showed that patellofemoral grafts appeared to fail earlier than grafts in the tibiofemoral compartment ( 70 vs. $79 \%$ at 10 years), but long-term survivorship was not significantly different ( 70 vs. $73 \%$ at 15 years). Concurrent osteotomy significantly $(p=0.01)$ increased graft survivorship. Survivorship was significantly $(p<0.001)$ lower in patients with a $>15 \mathrm{~cm}^{2}$ surface area transplanted. Patients younger than 30 years had a 15 -year survival rate of $84 \%$, significantly higher than patients aged 30 to 45 years $(p=0.02)$ and patients older than 45 years $(p=0.05)$.

Beris et $\mathrm{al}^{11}$ reported on 42 patients (mean defect size, $5.33 \mathrm{~cm}^{2}$ ) who underwent periosteum patch-covered ACI with a mean follow-up of 8 years. Patients older than 50 years, obese, or with kissing or multiple lesions were excluded. One patient was treated with MF 5 years after ACI because of graft delamination; in another patient, a partial detachment of graft was recorded 5 years after $\mathrm{ACl}$ and it was treated with regional trimming, removal of the detached segments, and subchondral MF. Numerous loose bodies, degeneration, and discoloration of the graft with histological features compatible with synovial chondromatosis were discovered in a 12-year-old girl after 8 months from ACI. Debridement of the graft site and MF for the treatment of the chondral defect and removal of all the loose bodies were performed.

In a multicenter study by Gomoll et $\mathrm{al}^{12}$ regarding treatment of patellofemoral chondral lesions with $\mathrm{ACI}$, a failure rate of $8.18 \%$ ( 9 patients) out of the 110 subjects (mean defect size, $5.4 \mathrm{~cm}^{2}$ ) treated with periosteum patch-covered ACI, was reported. Patients who required a concomitant trochlear graft were included; patients with defects outside the patellofemoral compartment were excluded. The authors considered a patient as failed with structural failure of the $\mathrm{ACI}$ diagnosed by magnetic resonance imaging (MRI) and/or arthroscopy in conjunction with pain requiring revision surgery. The mean follow-up was 7.5 years. Four of the nine failed patients were revised with TKR, four with partial knee replacement, and one patient declined further surgery.

Farr ${ }^{13}$ studied 38 subjects (mean defect size, $5.42 \mathrm{~cm}^{2}$ ) with full-thickness patellar and/or trochlear cartilage lesions and a median follow-up of 3.1 years. The author considered the ACI treatment as failed when patients had an operation after implantation that necessitated removal of the graft, confirmed partial or full delamination of the graft, confirmed a loss of defect fill, or violated the subchondral bone. The failure rate was $7.89 \%$ (three patients): two failed patients were treated by MF, whereas another patient had acute knee sepsis and the implants were removed.

Minas et al ${ }^{14}$ reported on 153 patients (155 knees; mean size of primary lesion, $6.7 \mathrm{~cm}^{2}$ ) with evidence of early osteoarthritis (peripheral intra-articular osteophyte formation and/or 0 to $50 \%$ joint space narrowing at X-rays or normal radiographs but evidence of kissing lesions or generalized chondromalacia noted at the time of surgery) treated with ACI (periosteal patch) and a mean follow-up of 5.35 years. Twelve of the 155 knees (8\%) were considered treatment failures and revised to partial (2) or total (10) joint arthroplasty at an average of 3.17 years after ACI. Subsequent surgical procedures (SSP) after the index implantation were performed in 95 of the 155 knees (61\%). In 21 knees, the indication for SSP was partial graft delamination affecting less than $20 \%$ of the defects, and treated with either MF (8), abrasion arthroplasty (five) or osteochondral autograft transfer system (OATS, 8). These patients were not classified as failure.

Jungmann et $\mathrm{al}^{15}$ analyzed a population of 413 patients (mean defect size, $5.6 \mathrm{~cm}^{2}$ ) with at least a 2-year follow-up after $\mathrm{ACI}$, of which 88 patients $(21.31 \%$ ) received reintervention characterized by an active manipulation at the transplantation site. One hundred nine patients were treated with periosteum patch-covered ACI, 235 patients with ChondroGide membrane (Geistlich, Wolhusen, Switzerland), and 69 patients with $\mathrm{MACI}$ (BioSeed-C). The time to revision surgery was 1.7 years for periosteum patch-covered $\mathrm{ACI}, 1.7$ years for Chondro-Gide-covered ACI, and 2.4 years for BioSeed-C treatment. At forward and backward binary logistic 
regression, female gender $(p=0.015)$, more than one previous surgery $(p<0.001)$, previous bone marrow stimulation (BMS, $p=0.017$ ), and periosteum patch-covered technique $(p=0.031)$ increased the risk for the need of reintervention.

Erggelet et al $^{16}$ compared two groups of patients treated by periosteum ACI (42 patients; mean defect size, $6.38 \mathrm{~cm}^{2}$ ) and BioSeed-C (40 patients; mean defect size, $4.6 \mathrm{~cm}^{2}$ ). In the periosteum group, 10 patients required reintervention due to symptomatic periosteal hypertrophy (4), graft failure (3), plica syndrome (2), and synovectomy (1). In the BioSeed-C group, reoperations were necessary and regarded as directly related to $\mathrm{ACI}$ in five patients. One patient underwent graft removal in a peripheral institution and was thus registered as a treatment failure. Two patients had a synovectomy and one patient had a debridement and one a TKR.

Niemeyer et al $^{17}$ published data $349 \mathrm{ACI}$ procedures on 309 patients (mean defect size, $4.6 \mathrm{~cm}^{2}$ ) with a mean followup of 5.5 years. Of the 349 procedures, 52 were performed with periosteum patch-covered technique, 215 with Chondro-Gide (Geistlich Biomaterials, Wolhusen, Switzerland) membrane-covered technique, and 82 with matrix-associated procedure (three-dimensional poly(lactic-co-glycolic) acid fleece). Revision surgery was performed in those cases in which patients had persistent pain and MRI revealed abnormal cartilage or subchondral bone signals in areas where ACI was performed. The mean time from initial ACI to the revision surgery was 1.7 years. Fifty-two patients (16.83\%) underwent revision surgery. Regarding the technical modification of ACI applied, the rate of patients treated with revision surgery was highest in the periosteum group (26.9\%), followed by the BioSeed-C group (14.6\%) and the Chondro-Gide-covered ACI group $(12.1 \%, p<0.008)$.

In the above mentioned randomized controlled clinical trial conducted by Bentley et $\mathrm{al}^{7}{ }^{7} 58$ patients (mean defect size, $4.41 \mathrm{~cm}^{2}$ ) with a minimum follow-up of 10 years were treated with ACI. Ten of the patients (17\%) resulted in a failed cartilage repair with a mean time from the index procedure to revision of 5.1 years. Regarding failure, ACI outperformed mosaicplasty (log-rank, $p=0.001$ ) and older ACI patients tended to fail earlier than younger ones $(p=0.028)$. Of the 10 failed patients, 1 underwent revision surgery with $\mathrm{ACI}, 3$ were revised with MACI, 1 was converted to UKR, 3 to TKR, 1 to PFJ replacement, and 1 underwent HTO.

Niethammer et $\mathrm{al}^{18}$ investigated the common revision operations after third-generation ACI (NOVOCART 3D; TETEC AG, Reutlingen, Germany) in a population of 143 consecutive patients (171 cartilage defects; mean defect size, $5 \mathrm{~cm}^{2}$ ) with a mean follow-up of 5 years. The revision rate was $22.4 \%$ $(n=32)$ with a mean time to revision of 1.15 years (15 MF/ drilling, 7 arthrolysis, $3 \mathrm{ACI}, 3$ arthroscopy, 2 retrograde drilling, 1 arthroplasty, and 1 HTO).

\section{Osteochondral Allograft Transplantation}

Briggs et $\mathrm{al}^{19}$ reported the results of OCA transplantation as primary treatment. Patients with any previous surgery on the affected knee were excluded from the study. Graft failure was defined as revision of the OCA or conversion to arthro- plasty. Of 55 patients (61 knees; mean graft size, $9.6 \mathrm{~cm}^{2}$; mean follow-up, 7.6 years) included in the study, 11 knees (18.03\%) were considered failure with a median time to failure of 3.5 years. Eight knees were converted to TKR, two knees had an OCA revision, and one knee had a patellectomy. The authors highlighted that six of nine knees (66.7\%) treated for degenerative chondral lesions failed. In five of these six knees, the location of the defect was the patellofemoral compartment. Furthermore, mean graft size in failed cases was larger than that in nonfailed ones (18.2 vs. $7.8 \mathrm{~cm}^{2}$ ).

Frank et $\mathrm{al}^{20}$ described a population of 180 patients (average of the largest osteoarthritis [OA] defect area, $5.6 \mathrm{~cm}^{2}$ ) who underwent OCA transplantation with a mean follow-up of 5.0 years. The failure criteria defined by authors were revision OCA transplantation, conversion to knee arthroplasty, or gross appearance of graft failure at secondlook arthroscopic surgery. Twenty-four patients (13\%) were considered failures at a mean of 3.6 years after the index OCA transplantation. These patients required revision OCA in 7 cases and conversion to arthroplasty in 12 cases. The other five grafts were considered failed because of appearance of a poorly incorporated allograft in arthroscopic surgery. In patients who were considered as failure, a higher body mass index (BMI, 29.42 vs. $26.00 \mathrm{~kg} / \mathrm{m}^{2} ; p=0.003$ ) and a significantly greater number of previous ipsilateral knee surgical procedures ( 3.75 vs. $2.28 ; p<.0001$ ) were recorded.

Levy et $\mathrm{al}^{21}$ evaluated 122 patients ( 129 knees; mean graft size, $8.1 \mathrm{~cm}^{2}$ ) who underwent isolated OCA transplantation of the femoral condyle; 13.5 years was the median follow-up of patients whose grafts were not surgically removed at the time of follow-up. The majority of knees had one or more surgeries before OCA transplantation, including cartilage repair surgery. The authors defined OCA failure as revision of the graft or conversion to partial or TKR. Based on these failure criteria, 31 knees (24\%) were classified as failure with a mean time to failure of 7.2 years. Fifteen failure patients underwent revision OCA transplantation, 13 were converted to TKA, and 3 to unicompartmental knee arthroplasty (UKA). Age and number of previous surgeries were associated with OCA failure after checking the other variables at the logistic regression.

Sadr et $\mathrm{al}^{22}$ analyzed the use of OCA transplantation to treat osteochondritis dissecans (OCD) of the knee. The study population was composed of 135 patients (149 knees; mean graft size, $7.3 \mathrm{~cm}^{2}$ ). The mean follow-up of patients with allograft in situ at last check was 6.3 years. Procedures that included removal of the allograft were considered as failure. Of the 12 knees (8\%), which were classified as OCA failures, 7 underwent revision OCA transplantation, UKA was performed in 3 cases, and TKA in 2 cases. The mean time to failure was 6.1 years.

Gracitelli et $\mathrm{al}^{23}$ reported data 27 patients (28 knees; mean graft size, $10.1 \mathrm{~cm}^{2}$ ) who had undergone an isolated OCA transplantation of the patella. A failure of the OCA transplantation was registered when reoperation resulted in removal of the allograft. Twenty of the 28 knees (71.4\%) had the allograft in situ at latest follow-up; the mean follow- 
up was 9.7 years. Eight of the 28 knees (28.6\%) were considered OCA failures and revised at a mean time of 1.45 years. Four were converted to TKA, two to PFJ replacement, one underwent revision OCA, and one underwent patellectomy.

In a population of 46 patients ( 48 knees; mean graft area, $19.2 \mathrm{~cm}^{2}$ ) who had undergone OCA transplantation for bipolar cartilage lesions of the knee, Meric et $\mathrm{al}^{24}$ reported that 22 of the 48 OCA transplantations (45.8\%) failed at a mean of 3.5 years. Failures included revision allografting, conversion to arthroplasty, arthrodesis, and patellectomy. Fourteen patients underwent TKA, 1 patient UKA, 1 PFJ replacement, and 2 arthrodesis. One of the three patients who repeated OCA transplantation was treated with patellectomy after traumatic fall. The mean follow-up of 26 knees with grafts still in situ was 7 years. The allograft size in the failure group was significantly larger when compared with that in the nonfailure group.

Murphy et $\mathrm{al}^{25}$ reported the outcome of OCA transplantation in a population younger than 18 years at the time of surgery. Thirty-nine patients (43 knees; mean graft area, 8.4 $\mathrm{cm}^{2}$ ) with a mean follow-up of 8.4 years were included in the study. OCD was noted as the most common cause of the lesions (61\%). The authors defined the need for OCA revision or conversion to arthroplasty as failure. Five knees (12\%) experienced a failure of the allograft at a median of 2.7 years after OCA transplantation. All failed allografts were treated with OCA revision.

In the population studied by Degen et $\mathrm{al}^{9}{ }^{9}$ a group of 14 patients (mean defect size, $5.92 \mathrm{~cm}^{2}$ ) older than 40 years underwent OCA transplantation for a symptomatic distal femoral cartilage lesion. Six patients (43\%) required further surgery at an average of 5.14 years; four underwent arthroscopic debridement, lysis of adhesions, and removal of loose bodies. The other two patients underwent revision cartilage procedures, namely AOT and OCA revisions.

Sterilized and Decellularized Osteochondral Allograft Farr et $\mathrm{al}^{26}$ reported the high failure rate of a sterilized and decellularized osteochondral allograft (SDOCA) for the treatment of cartilage defects. Thirty-two patients (mean defect size, $2.9 \mathrm{~cm}^{2}$ ) with a mean follow-up of 1.29 years and up to two full-thickness cartilage lesions of the knees treated with SDOCA implant (Chondrofix; ZimmerBiomet) were evaluated. The failure criteria established by the authors included any reoperation resulting in removal of the implant, and/or when MRI or arthroscopy showed evidence of subchondral collapse or loss of more than $50 \%$ of the articular cartilage cap of a plug. Twenty-three of the 32 knees (72\%) were considered treatment failures. After failure, eight patients were treated with OCA transplantation, three with TKA, and one with UKA. The statistical analysis demonstrated that age was a significant predictor of failure.

\section{Synthetic Scaffolds}

Dhollander et $\mathrm{al}^{27}$ reported the outcome of 20 subjects (mean defect size, $0.83 \mathrm{~cm}^{2}$ ) with 1 focal cartilage defect involving the femoral condyle, patella, or trochlea treated with osteochondral scaffold plugs (TruFit plug, Smith \& Nephew, Andover, Massachusetts, United States). Fifteen of them completed 1 year of follow-up, out of which three (20.0\%) were considered as clinical failures and therefore were eligible for revision surgery. The subsequent osteochondral defect was filled with autologous bone grafts harvested from the iliac crest.

Joshi et $\mathrm{al}^{28}$ described a population of 10 patients (mean defect size, $2.64 \mathrm{~cm}^{2}$ ) affected by patellar full-thickness chondral defects treated by osteochondral scaffold plugs (TruFit plug, Smith \& Nephew, Andover, Massachusetts, United States). Seven patients (70\%) were revised, two patients were converted to patellar arthroplasty, and five patients were treated with implant removal, bone filling of the defect, marrow stimulation of the surrounding bone, and fibrin coating on the surface.

In the previous described study of Degen et al, ${ }^{9}$ in a population of 61 patients older than 40 years and affected by distal femoral cartilage lesions involving the medial or lateral femoral condyle, 35 (mean defect size, $3.24 \mathrm{~cm}^{2}$ ) were treated with osteochondral scaffold plugs (TruFit plug, Smith \& Nephew, Andover, Massachusetts, United States). Four patients $(11.4 \%)$ required revision at an average of 3.15 years, two underwent AOT, while the other two were converted to TKA.

Berruto et $\mathrm{al}^{29}$ described the use of MaioRegen (FinCeramica Faenza SpA, Faenza, Italy) in the treatment of large knee osteochondral lesions. They enrolled 49 patients in the study (mean defect size, $4.35 \mathrm{~cm}^{2}$ ). All patients were followed prospectively with a minimum follow-up of 2 years. The treatment failed in five patients, one patient underwent UKR, one sustained a HTO, one patient was treated with autologous osteochondral transplantation, and another one with OCA transplantation and concomitant distal femoral osteotomy (DFO). The last failure continued the treatment in another institution.

\section{Treatment of Failed Cartilage Repair}

Gracitelli et $\mathrm{al}^{30}$ analyzed a population of 163 patients (164 knees; mean graft size, $6.8 \mathrm{~cm}^{2}$ ) treated with OCA transplantation after previous failed cartilage repair. Patients had a minimum follow-up of 2 years (mean of 8.5 in subjects whose grafts were still in situ). SMS was the most common isolated previous procedure (88.4\%). The authors considered any reoperation resulting in removal of the graft as failure. The authors reported an improvement in pain and function from preoperatively to the latest follow-up in all functional scores used (modified Merle d' Aubigné-Postel [18-point], the international knee documentation committee [IKDC] score, the Knee Injury and Osteoarthritis Outcome Score [KOOS], and Knee Society Function [KS-F] score). Sixty-eight knees (41.5\%) had reoperations after OCA transplantation and 31 (19.02\%) were considered failures. Of the 164 knees, 18 failed knees were converted toTKA, 9 had an OCA revision, 2 were converted to UKA, 1 to PFJ arthroplasty, and 1 to arthrodesis. The median time to failure was 2.6 years. Survivorship of the OCA transplantation was 87.8 and $82 \%$ at 5 and 10 years, respectively. Failure patients had a 
significantly increased BMI and number of previous surgeries compared with nonfailure patients.

In another study, Gracitelli et $\mathrm{al}^{31}$ compared two groups of patients who underwent OCA transplantation. A consecutive series of 46 knees that had OCA transplantation performed as a primary treatment (group 1) were matched to a nonconsecutive series of 46 knees that underwent OCA transplantation after failure of previous subchondral marrow stimulation (group 2). Any reoperation resulting in removal of the graft was defined as failure. In group 1, 11 of 46 knees (24\%) underwent reoperations, and in group 2, 20 of 46 knees (44\%) underwent reoperations. A significant difference in reoperation rate was found between the groups $(p=0.04)$. Failure OCA transplantation was noted in five knees (11\%) in group 1; two knees had the OCA revised and the other three were converted to TKR. In group 2, seven knees (15\%) were considered failures, of which three had the OCA revised and four were converted to TKR. The failure rate was not statistically significant between the groups $(p=0.53)$. Similar survivorship of the allograft was found at 10-year followup; $87.4 \%$ of group 1 and $86 \%$ of group 2. Patients whose grafts remained in situ had a mean follow-up of 7.8 years (41 knees in group 1) and 11.3 years (39 knees in group 2). Improvement in pain and function from the preoperative point to the latest follow-up was registered in both the groups ( $p<0.001)$. No difference between the groups was found preoperatively, at the latest follow-up, or in the change from preoperative state to follow-up.

In the study of Frank et al, ${ }^{20}$ Kaplan-Meier survival analysis was performed on a population of 180 patients treated with OCA transplantation, and no significant difference in survival distributions of OCA transplantation between patients with and without a history of previous MF was registered at log-rank test $(p=0.370)$.

Horton et $\mathrm{al}^{32}$ reported data of 33 knees (mean graft area, $9.5 \mathrm{~cm}^{2}$ ) that underwent revision OCA transplantation of the knee after failure of primary OCA. All included patients had undergone surgery at least 2 years ago with a minimum follow-up of 2 years. The mean follow-up after receiving a revision OCA transplantation was 10 years. The authors classified any patient as revision allograft failure who underwent conversion to partial or TKA. Thirteen patients (39\%) underwent graft-related surgery and were considered failures. Of these, 1 was converted to UKA and 12 were converted to TKA with a mean time to failure of 5.5 years. Revision graft survivorship was 79 and $61 \%$ at 5 and 10 years, respectively. The authors reported an increased incidence of failure and conversion to partial or TKA in older patients, patients with diagnosis of $\mathrm{OA}$, and those who had a progression of cartilage disease at the time of revision OCA transplantation. However, sample size was too small to assess which variables predicted allograft failure.

Murphy et $\mathrm{al}^{25}$ reported that 5 of 43 knees (12\%) failed in their study population composed by subjects younger than 18 years who underwent OCA transplantation. These failures were treated by OCA revision. Of these five grafts, four (80\%) were still in situ at latest follow-up (median, 4.5 years) with clinical scores similar to the entire cohort. One patient underwent knee replacement 8.6 years after the revision allograft.

Stone et $\mathrm{al}^{33}$ evaluated the effect of OAT on previously failed surgical repair of OCD lesions. Seven patients were enrolled in the study (mean defect size, $3.26 \mathrm{~cm}^{2}$; mean follow-up of 7 years). A significant improvement from preoperative IKDC, the Western Ontario and McMaster Universities Osteoarthritis Index (WOMAC), and Tegner score to most recent follow-up was recorded.

Vijayan et $\mathrm{al}^{34}$ described their experience with revision cartilage surgery in patients with isolated chondral or osteochondral defect after failed ACI or MACI. The study population was composed of 22 patients (mean defect size, $4.46 \mathrm{~cm}^{2}$ ) with a mean follow-up of 5.4 years. The primary surgery was $\mathrm{ACI}$ in 17 and $\mathrm{MACI}$ in 5 patients. Revision surgeries were $13 \mathrm{MACl}$ performed with chondrocyteseeded porcine type I/III collagen membrane, and $9 \mathrm{ACI}$ with use of Chondro-Gide type I/III collagen membrane (Geistlich Biomaterials, Wolhusen, Switzerland) or periosteum. The failure criteria adopted by the authors included a persistent pain with a visual analogue scale (VAS) equal to or higher than preoperatively documented, with associated "poor" functional scores (modified Cincinnati knee score, Stanmore-Bentley score, VAS for pain), and arthroscopic or radiological confirmation of graft failure. All three clinical outcome scores following revision cartilage transplantation showed a significant improvement. One patient reported knee pain after 3 years from revision surgery with arthroscopic confirmation of graft delamination. The patient was treated with a third revision MACI procedure for a lesion of the patella, resulting in an "excellent" modified Cincinnati knee score, VAS of zero, and a Stanmore-Bentley score of zero at latest follow-up of 6 years. Pain and intermittent locking of the knees was recorded in two patients. At arthroscopy, graft delamination of their patella lesions was confirmed. Both underwent patellofemoral joint replacement at 14 and 25 months subsequent to the revision surgery.

In the previously mentioned population described by Minas et $\mathrm{al}^{10} 89$ of 210 (42.2\%) patients treated with periosteum-covered ACI had earlier undergone SMS. The authors reported that survivorship of ACI was significantly lowered $(p=0.004)$. In patients with successful ACI, no difference was found in clinical outcome scores between knees previously treated with marrow stimulation and those in which $\mathrm{ACI}$ was the primary cartilage repair procedure.

Jungmann et al $^{15}$ analyzed a population of 413 patients treated with $\mathrm{ACI}$, of which 88 patients had reintervention. One hundred twenty-four $(30.02 \%)$ patients had at least one previous cartilage treatment at the same location of the knee joint, mainly MF, Pridie drilling, or previous ACI. The binary logistic regression showed an increased risk for the need of reintervention characterized by an active manipulation at the transplantation site in patients with previous SMS $(p=0.017)$.

In the study population evaluated by Niethammer et al, ${ }^{18}$ of the 143 patients with 171 defects treated with third generation ACI (NOVACART 3D, TETEC AG, Reutlingen, 
Germany), 34 (19.9\%) of these defects had previous failed cartilage repair procedures. At statistical analysis, cartilage defects with second-line therapy after a previously performed cartilage repair before $\mathrm{ACI}$, showed no higher complication or revision rate.

\section{Discussion}

From the analysis of the selected studies, it is possible to observe that even if used to treat small chondral defects, failure rate of MF is not negligible. Furthermore, MF showed a short time to failure or revision. ${ }^{4,5}$ Regarding possible risk factors, Salzmann et al reported that the risk of failure is higher in patient with history of a previous surgery to the knee, and curiously, in patients with smaller lesions and smaller defect areas per knee. ${ }^{4}$ Even if based on a small sample size and in absence of a statistical analysis, the results reported by Steadman et al suggest that MF technique may be an acceptable treatment in adolescent patients with small cartilage defects. It is interesting to note that in their adolescent population, the three recorded failures were in female patients treated for patellofemoral defects. ${ }^{6}$

In the RCT of Bentley et al, OAT (mosaicplasty) led to revision surgery in more than $50 \%$ of the cases in approximately 4 years. ${ }^{7}$ Better results were showed in mid-term follow-up in patients with small cartilage defects. ${ }^{8,9}$ Based on the results of Degen et al, the use of mosaicplasty in patients older than 40 years with focal cartilage defects but with untreated regions of the knee no worse than Outerbridge grade 2 , may be a viable options at short-/mid-term. ${ }^{9}$

ACI provides satisfactory results in terms of both pain relief and knee function rehabilitation in patients with large full-thickness chondral and osteochondral defects of the knee. $^{35}$ In the study of Minas et al, ${ }^{14}$ the failure rate of periosteum-covered $\mathrm{ACI}$ was $25 \%$. It is important to highlight that this result was recorded in a population with a minimum follow-up of 10 years. Furthermore, the total surface area treated per knee was $8.4 \mathrm{~cm}^{2}$ and the authors considered patients with graft in situ but with development of new defects elsewhere in the same knee as failure, which required further surgery. Combined HTO, defect size $<15 \mathrm{~cm}^{2}$ and age $<30$ years are related to a higher survivorship. ${ }^{10}$ Bentley et al reported that older patients tend to fail earlier than younger patients. ${ }^{7}$ Beris et $\mathrm{al}^{11}$ reported that only 3 patients required a subsequent revision cartilage repair surgery on a population of 42 patients treated with periosteum patchcovered ACI. ${ }^{11}$ In patellofemoral compartment, periosteumcovered ACI showed a good survivorship at short ${ }^{13}$ and midlong term. ${ }^{12}$ Interestingly, the paper of Minas et al $^{14}$ reports that in patients with early $\mathrm{OA}$, periosteum covered $\mathrm{ACI}$ seems to have a failure rate similar to those registered in other studies, but this data may be in part related to the different failure criteria used by the authors. ${ }^{14}$ In a large cohort of patients treated with ACI (first, second, and third generation), $21.3 \%$ needed an active reintervention at the transplant site. Increased risk of reintervention was registered in the presence of the following factors: female gender, more than one previous surgery, previous BMS, and periosteum- covered $\mathrm{ACI} .{ }^{15} \mathrm{~A}$ higher revision rate in patients who underwent periosteum-covered ACI was also reported in other studies. ${ }^{16,17}$ In the paper of Niethammer et $\mathrm{al},{ }^{18}$ third generation autologous chondrocyte implantation (NOVACART 3D, TETEC AG, Reutlingen, Germany) showed a short time to revision surgeries ( 1.15 years) even if not all surgeries were related to active manipulation at implant site or to implant removal. $^{18}$

The use of OCA transplantation in a population without previous cartilage repair surgery showed a failure rate of $18 \%$ at a median time of 3.5 years. Degenerative chondral lesions, larger graft size, and patellofemoral location are probably related to an increased risk of failure. ${ }^{19}$ In the large cohort of patients described by Frank et $\mathrm{al}^{20}$ without history of previous knee surgeries as exclusion criteria, a similar failure rate and time to failure was recorded (13\%, 3.6 years) ${ }^{20} \mathrm{At}$ long-term follow-up the failure rate of OCA transplantation obviously increased (24\%), but contemporary, a long mean time to failure was reported (7.2 years). ${ }^{21}$ Higher BMI, ${ }^{20}$ age, ${ }^{21}$ and a greater number of previous ipsilateral knee surgeries seemed to increase the risk of failure. ${ }^{20,21}$ OCA transplantation in the treatment of OCD showed a low rate of failure with a time to failure of approximately 6 years. ${ }^{22}$ Regarding treatment of chondral defects of the patella, the failure rate seemed to be higher and the mean time to revision shorter. ${ }^{23}$ The same results were reported for bipolar cartilage lesions. In this case, a larger allograft size showed correlation to a higher failure rate. ${ }^{24}$ Murphy et $\mathrm{al}^{25}$ reported that in patients younger than 18 years, OCA transplantation can be considered a valuable treatment for large symptomatic osteochondral lesions in the knee (12\% of failure rate in a population with a mean follow-up of 8.4 years). ${ }^{25}$

SDOCA implants showed a very high failure rate. Furthermore, this result was recorded in a short follow-up time. ${ }^{26}$ The use of TruFit plug for the treatment of small cartilage defects seemed to be related to a higher failure rate at shortterm, ${ }^{27}$ especially if used to treat patellar defects. ${ }^{28}$ Conversely, the treatment of osteochondral lesions with MaioRegen showed promising results in terms of survivorship at short term. ${ }^{29}$

Regarding the treatment of failed cartilage repair, the use of OCA transplantation in patients with previous failed cartilage repair may be a safe option. In this situation, OCA showed a failure rate similar to that reported in general population and significant improvement of functional scores from preoperative condition. After a failed cartilage repair, patients treated by OCA transplantation with higher BMI and a greater number of previous surgeries seemed to have an increased risk of failure. ${ }^{30}$ In patients with previous failed SMS, compared with those with no previous cartilage repair, OCA transplantation showed a statistically significant rate of reoperation but not of failure. At long-term follow up, the survivorship was similar. ${ }^{20,31}$ In clinical and functional scores, patients with previous failed SMS demonstrated an improvement similar to patients treated with OCA transplantation as primary treatment. ${ }^{31}$ Even if these results were derived from a small size sample, the revision of failed OCA 
transplantation with further OCA seemed to have a greater failure rate compared with revision of a previous cartilage repair surgery in general. In these patients, older age, diagnosis of OA, or progression of cartilage disease at the time of revision may be correlated with an increased incidence of failure. ${ }^{32}$ In younger patients, revision OCA after failed OCA transplantation may have better results, but this hypothesis should be proved in more specific studies. ${ }^{25}$ Patients with previous failed $\mathrm{ACI}$ or MACI who underwent further MACI or $\mathrm{ACI}$ reported acceptable functional and survivorship results. It is interesting to note that in a small population of 22 patients treated with revision $\mathrm{MACI}$ or $\mathrm{ACI}$, all 3 patients who required third revision $\mathrm{MACI}$ or partial replacement had a patellar cartilage defect. ${ }^{34}$ Otherwise, $\mathrm{ACI}$ in patients with a history of previous SMS demonstrated a greater failure rate. ${ }^{10,15}$ An explanation for this difference could be the hypothesis that SMS worsens the microarchitecture of the underlying bone for inevitable future surgical intervention, ${ }^{36,37}$ and in this case, OCA transplantation should probably be preferred. Third generation ACI used in 34 patients with previous failed cartilage repair did not demonstrate an increased failure rate compared with patients treated with third generation ACI as first-line therapy. ${ }^{18}$ This analysis has several limitations. First, we limited our search to studies published in the last 10 years to minimize bias related to temporal changes in cartilage repair surgery. This choice may have excluded significant studies. Second, we did not perform a quantitative analysis due to two main reasons: the limited number of cases and the heterogeneity of the definition of failed cartilage repair among different papers.

In conclusion, management of failed cartilage repair depends on the type of failed surgical treatment. OCA transplantation seems to be the most reliable treatment, especially in the presence of a previous failed SMS. ACI or MACI showed acceptable results in patients with previously failed MACI or ACI, but this hypothesis should be confirmed by further studies. According to the findings of this review, we retain that great attention should be paid to the condition of the subchondral bone when approaching a patient with history of failed cartilage repair.

\section{References}

1 Curl WW, Krome J, Gordon ES, Rushing J, Smith BP, Poehling GG. Cartilage injuries: a review of 31,516 knee arthroscopies. Arthroscopy 1997;13(04):456-460

2 Biant LC, McNicholas MJ, Sprowson AP, Spalding T. The surgical management of symptomatic articular cartilage defects of the knee: consensus statements from United Kingdom knee surgeons. Knee 2015;22(05):446-449

3 Bedi A, Feeley BT, Williams RJ III. Management of articular cartilage defects of the knee. J Bone Joint Surg Am 2010;92(04):994-1009

4 Salzmann GM, Sah B, Südkamp NP, Niemeyer P. Reoperative characteristics after microfracture of knee cartilage lesions in 454 patients. Knee Surg Sports Traumatol Arthrosc 2013;21(02): 365-371

5 Solheim E, Øyen J, Hegna J, Austgulen OK, Harlem T, Strand T. Microfracture treatment of single or multiple articular cartilage defects of the knee: a 5-year median follow-up of 110 patients. Knee Surg Sports Traumatol Arthrosc 2010;18(04):504-508
6 Steadman JR, Briggs KK, Matheny LM, Guillet A, Hanson CM, Willimon SC. Outcomes following microfracture of full-thickness articular cartilage lesions of the knee in adolescent patients. J Knee Surg 2015;28(02):145-150

7 Bentley G, Biant LC, Vijayan S, Macmull S, Skinner JA, Carrington RW. Minimum ten-year results of a prospective randomised study of autologous chondrocyte implantation versus mosaicplasty for symptomatic articular cartilage lesions of the knee. J Bone Joint Surg Br 2012;94(04):504-509

8 Robb CA, El-Sayed C, Matharu GS, Baloch K, Pynsent P. Survival of autologous osteochondral grafts in the knee and factors influencing outcome. Acta Orthop Belg 2012;78(05):643-651

9 Degen RM, Coleman NW, Chang B, Tetreault D, Mahony GT, Williams RJ. Outcomes following structural grafting of distal femoral osteochondral injuries in patients aged 40 years and older. J Knee Surg 2017;30(03):244-251

10 Minas T, Von Keudell A, Bryant T, Gomoll AH. The John Insall Award: a minimum 10-year outcome study of autologous chondrocyte implantation. Clin Orthop Relat Res 2014;472(01):41-51

11 Beris AE, Lykissas MG, Kostas-Agnantis I, Manoudis GN. Treatment of full-thickness chondral defects of the knee with autologous chondrocyte implantation: a functional evaluation with long-term follow-up. Am J Sports Med 2012;40(03):562-567

12 Gomoll AH, Gillogly SD, Cole BJ, et al. Autologous chondrocyte implantation in the patella: a multicenter experience. Am J Sports Med 2014;42(05):1074-1081

13 Farr J. Autologous chondrocyte implantation improves patellofemoral cartilage treatment outcomes. Clin Orthop Relat Res 2007; 463:187-194

14 Minas T, Gomoll AH, Solhpour S, Rosenberger R, Probst C, Bryant T. Autologous chondrocyte implantation for joint preservation in patients with early osteoarthritis. Clin Orthop Relat Res 2010; 468(01):147-157

15 Jungmann PM, Salzmann GM, Schmal H, Pestka JM, Südkamp NP, Niemeyer P. Autologous chondrocyte implantation for treatment of cartilage defects of the knee: what predicts the need for reintervention? Am J Sports Med 2012;40(01):58-67

16 Erggelet C, Kreuz PC, Mrosek EH, et al. Autologous chondrocyte implantation versus ACI using 3D-bioresorbable graft for the treatment of large full-thickness cartilage lesions of the knee. Arch Orthop Trauma Surg 2010;130(08):957-964

17 Niemeyer P, Pestka JM, Kreuz PC, et al. Characteristic complications after autologous chondrocyte implantation for cartilage defects of the knee joint. Am J Sports Med 2008;36(11): 2091-2099

18 Niethammer TR, Valentin S, Ficklscherer A, Gülecyüz MF, Pietschmann MF, Müller PE. Revision surgery after third generation autologous chondrocyte implantation in the knee. Int Orthop 2015;39(08):1615-1622

19 Briggs DT, Sadr KN, Pulido PA, Bugbee WD. The use of osteochondral allograft transplantation for primary treatment of cartilage lesions in the knee. Cartilage 2015;6(04):203-207

20 Frank RM, Lee S, Levy D, et al. Osteochondral allograft transplantation of the knee: analysis of failures at 5 years. Am J Sports Med 2017;45(04):864-874

21 Levy YD, Görtz S, Pulido PA, McCauley JC, Bugbee WD. Do fresh osteochondral allografts successfully treat femoral condyle lesions? Clin Orthop Relat Res 2013;471(01):231-237

22 Sadr KN, Pulido PA, McCauley JC, Bugbee WD. Osteochondral allograft transplantation in patients with osteochondritis dissecans of the knee. Am J Sports Med 2016;44(11):2870-2875

23 Gracitelli GC, Meric G, Pulido PA, Görtz S, De Young AJ, Bugbee WD. Fresh osteochondral allograft transplantation for isolated patellar cartilage injury. Am J Sports Med 2015;43(04):879-884

24 Meric G, Gracitelli GC, Görtz S, De Young AJ, Bugbee WD. Fresh osteochondral allograft transplantation for bipolar reciprocal osteochondral lesions of the knee. Am J Sports Med 2015; 43(03):709-714 
25 Murphy RT, Pennock AT, Bugbee WD. Osteochondral allograft transplantation of the knee in the pediatric and adolescent population. Am J Sports Med 2014;42(03):635-640

26 Farr J, Gracitelli GC, Shah N, Chang EY, Gomoll AH. High failure rate of a decellularized osteochondral allograft for the treatment of cartilage lesions. Am J Sports Med 2016;44(08): 2015-2022

27 Dhollander AA, Liekens K, Almqvist KF, et al. A pilot study of the use of an osteochondral scaffold plug for cartilage repair in the knee and how to deal with early clinical failures. Arthroscopy 2012;28(02):225-233

28 Joshi N, Reverte-Vinaixa M, Díaz-Ferreiro EW, Domínguez-Oronoz R. Synthetic resorbable scaffolds for the treatment of isolated patellofemoral cartilage defects in young patients: magnetic resonance imaging and clinical evaluation. Am J Sports Med 2012;40(06):1289-1295

29 Berruto M, Delcogliano M, de Caro F, et al. Treatment of large knee osteochondral lesions with a biomimetic scaffold: results of a multicenter study of 49 patients at 2-year follow-up. Am J Sports Med 2014;42(07):1607-1617

30 Gracitelli GC, Meric G, Pulido PA, McCauley JC, Bugbee WD. Osteochondral allograft transplantation for knee lesions after failure of cartilage repair surgery. Cartilage 2015;6(02):98-105
31 Gracitelli GC, Meric G, Briggs DT, et al. Fresh osteochondral allografts in the knee: comparison of primary transplantation versus transplantation after failure of previous subchondral marrow stimulation. Am J Sports Med 2015;43(04):885-891

32 Horton MT, Pulido PA, McCauley JC, Bugbee WD. Revision osteochondral allograft transplantations: do they work? Am J Sports Med 2013;41(11):2507-2511

33 Stone KR, Pelsis JR, Crues JV III, Walgenbach AW, Turek TJ. Osteochondral grafting for failed knee osteochondritis dissecans repairs. Knee 2014;21(06):1145-1150

34 Vijayan S, Bentley G, Rahman J, Briggs TW, Skinner JA, Carrington RW. Revision cartilage cell transplantation for failed autologous chondrocyte transplantation in chronic osteochondral defects of the knee. Bone Joint J 2014;96-B(01):54-58

35 Rosa D, Balato G, Ciaramella G, Soscia E, Improta G, Triassi M. Long-term clinical results and MRI changes after autologous chondrocyte implantation in the knee of young and active middle aged patients. J Orthop Traumatol 2016;17(01):55-62

36 Bert JM. Abandoning microfracture of the knee: has the time come? Arthroscopy 2015;31(03):501-505

37 Zedde P, Cudoni S, Giachetti G, et al. Subchondral bone remodeling: comparing nanofracture with microfracture. an ovine in vivo study. Joints 2016;4(02):87-93 